\title{
Understanding emotional and psychological harm of people with intellectual disability: an evolving framework
}

\author{
Sally Robinson and Lesley Chenoweth
}

Sally Robinson and Lesley Chenoweth are based in the School of Human Services and Social Work, Griffith University, Brisbane, Australia.
At the time of writing Sally Robinson was a Research Fellow based at The School of Human Services and Social Work, Griffith University, Australia. She is now a postdoctoral fellow at Southern Cross University, Australia.

\begin{abstract}
Purpose - A schema for more clearly understanding the emotional and psychological abuse and neglect of people with intellectual disability was developed to support a narrative study with people with intellectual disability, families and other supporters about the lived experience of this maltreatment in disability accommodation services in Australia. This paper aims to describe the review of emotional and psychological abuse and neglect and the evolving new framework.

Design/methodology/approach - A review of existing understandings of this form of abuse in research and policy was conducted, and a framework developed and tested for "trustworthiness" with participants in the research.

Findings - A framework of emotional and psychological abuse and neglect is presented. It centres on the misuse of power and control, and goes into some detail in describing the sorts of behaviours and interactions which can occur when it is inflicted, and is tested against the experiences of people who have experienced this sort of abuse and neglect.

Research limitations/implications - This is an evolving framework, applied through one study only. Further application and research is needed to test the robustness of the framework.

Originality/value - A more complex construction of emotional and psychological abuse and neglect may inform the development of service policy and support education for people with disability, families, and workers.

Keywords Emotional abuse, Psychological abuse, Intellectual disability, Definition, Accommodation services, Abuse framework, Mental health services, Australia, Disabilities

Paper type General review

\section{Introduction}

The views of people with intellectual disability about their experiences of emotional and psychological abuse and neglect feature very little in either the disability studies or abuse literatures. This paper draws from a narrative study which aimed to find out about these experiences, working with people with intellectual disability, their families and other supporters to develop a detailed picture of the emotional and psychological abuse and neglect experienced by nine people whilst living in disability accommodation services in Australia. While not reporting on the outcomes of the research, this article centres on the issues of terminology we faced in defining this maltreatment, and our response to these challenges.

We briefly canvass existing conceptions of emotional and psychological abuse and neglect in the disability studies and related literatures, and in policy, with a particular focus on the Australian context. Following this is a description of the development of an emotional and psychological abuse and neglect framework which somewhat fleshes out this form


of maltreatment. We conclude with a discussion of implications for research, policy and practice arising from the use of the framework in this context.

\section{Background}

Emotional and psychological abuse and neglect remains a source of debate in both research and policy. It is undoubtedly harder to identify than other forms of mistreatment. No injury can be seen, unless the person has also undergone physical or sexual assault (Penhale, 1999; McKinnon, 2008). The impact of emotional and psychological abuse, while potentially severe, may be cumulative, reliant on a sustained attack on the person's psyche (Iwaniec et al., 2006). Unlike sexual or physical assault, emotional and psychological abuse may be unintentional on the part of the abuser (as in the case of an undertrained staff member following an unnecessarily restrictive behaviour management plan). It is also difficult to isolate from other forms of abuse because other forms of abuse also include emotional or psychological harm (Sobsey, 1994; Kairys et al., 2002). Some authors argue that it can be more strongly associated with the domain of omission whereas most other forms of abuse and assault reside within the domain of commission, or action - so neglect may have more complex role to play than in other abuse areas (Glaser, 2002; Sobsey, 1994).

\section{Underpinning contextual features: misuse of power and control}

Abuse of people with disability who live in disability accommodation services happens within a particular cultural and environmental context. Research by Marsland et al. (2007) sought to identify aspects of intellectual disability service cultures and environments, which could act as early indicators that people with intellectual disability were at risk of abuse. They found that the behaviours, actions, attitudes and decisions of managers and staff, the behaviours of people with intellectual disability, isolation, service design, placement planning and commissioning, and fundamental care and the quality of the environment were all critical in preventing a climate in which abuse could flourish. They also found that power, choice and the organisational climate were significant issues, noting that:

power is a significant construct underlying the issue of abuse. Specific issues relating to power have been associated with cultures of abuse. Where there are imbalances of power at any level within the care hierarchy, there is a risk that power will be used inappropriately, increasing the risk of abuse (Marsland et al., 2007, p. 12).

It is argued that there is a serious imbalance of power structured into traditional disability services, which perpetuates a climate that is ripe for abuse. Mandeville and Hanson (2000, p. 15) contend that this power imbalance is pervasive and that it "permeates program policy, agency culture, professional practice, and the personal dynamics in paid relationships". Ticoll (1994) details some of the ways in which people with disability are less powerful than those who abuse them. In some situations, people are reliant on perpetrators for physical, psychological or economic support or other necessities, they do not have control over the actions of others who have the potential to invade or disrupt their lives or well-being, and they lack credibility or "voice" if they have the opportunity to make a complaint about their mistreatment.

According to the seminal work of Wardhaugh and Wilding (1993) on the corruption of care, a number of organisational factors are in play which can result in the conception of people using services as less than fully human - including the neutralisation of normal moral concerns, isolation, a failure of management and lack of accountability and an enclosure of the organisation from scrutiny. Failure to address the fundamental imbalance of power leaves people with intellectual disability vulnerable to the potential abuse of individuals and systems, as they have little recourse to change their own situations.

There is clearly not a symmetry of power and control between people who use services and people who provide them. At times, there is a need for other people to take control - for example, when dealing with significant health issues such as epilepsy. More frequently, there is a difficult middle ground to navigate, for instance around the need of people for 
support and practical strategies to address challenging behaviour. Structured programmes and effective teaching which help people to develop practical and relational skills which are in the long-run protective can involve the ceding, or taking, of power and control. It is very important, in the context of abuse prevention and response, to ensure that safeguards and protections are in place around individual interactions and relationships to ensure that a climate which is preventative of abuse is cultivated, and that when problems do arise, that abuse is recognised and quickly stopped. Mechanisms which dovetail on several levels person-centred circles of support, positive behaviour support plans, service evaluation and monitoring and training and education, for example - need to be engaged to ensure that maltreatment is picked up on multiple levels, and responded to in varying ways.

\section{Institutional abuse}

Institutional abuse is a relevant concept to inform this framework, given that it focuses explicitly on people's experiences in formal environments of care. Two conceptions of institutional abuse have arisen. The first centres around place - the institution itself. The second centres around practices and cultures. For Sobsey (1994, pp. 91-93), there are four factors which make institutional abuse unique - extreme power inequities between residents and staff; the collective nature of the abuse; the abuse is covered up, or the knowledge of it is not shared outside the institution; and there are clearly defined patterns of environmental influence. Brown (2007, p. 2) understands institutional abuse to be:

not really a "type" of abuse, or even just a "site" of abuse, but a constellation of factors that interact to produce poor care, insensitive practice and to either provoke or condone individual or collective acts of cruelty.

The sorts of practices which occur within this conception of institutional abuse include the development and maintenance of poor quality environments, oppressive routines, neglect of the needs, preferences and aspirations of people living in the service, practices which are outside of community norms, individual and group cruelty and negligence.

\section{Conceptions of emotional and psychological abuse and neglect in the literature}

Much of the disability literature includes reference to emotional and psychological abuse as one of many forms of abuse. Women with Disabilities Australia (WWDA), in their global review of violence against women with disabilities, draw from the work of many researchers to define emotional or psychological violence as:

the infliction of anguish, pain, or distress through verbal or non-verbal acts and/or behaviour. It results in harm to a person's self-concept and mental well-being as a result of being subjected to behaviours such as verbal abuse, continual rejection, withdrawal of affection, physical or social isolation and harassment, or intimidation (WWDA, 2007, p. 33).

Other definitions include reference to lack of love and affection, corrupting, belittling, threats, verbal attacks, taunting and shouting which leads to the victim's loss of confidence and self-esteem, and omissions of care that produce harm (Westcott, 1993; Ticoll, 1994; Nosek et al. , 2001b; Conway, 1994). Saxton (2009, pp. 3-4) takes an interesting approach, and rejects an incident based notion of abuse. She and her colleagues at the World Institute on Disability state:

We regard abuse as a continuum of systematic mistreatment, extreme or subtle, the visible version of disability oppression.

Neglect is represented in two ways in the literature. One conception is broadly as a form of abuse (as is sexual abuse, physical abuse, financial abuse and so on), which occurs in the lives of people with intellectual disability, not just in their emotional and psychological lives (Horne et al., 2001; Nosek et al., 2001a; Kovener, 2000; Ticoll, 1994).

The second conception of neglect in the disability literature is less common, where neglect is presented as a more specific form of emotional and psychological neglect (Jenkins and Davies, 2006; Conway et al., 1996). Sobsey (1994, p. 34) is one of the few writers who specifically discusses emotional neglect in the literature. He states that: 
Neglect is perhaps the most insidious form of abuse; in extreme form it may be one of the most damaging ... Emotional or developmental neglect occurs when an individual is deprived of basic human interactions required for the development of normal behaviour.

\section{Emotional and psychological abuse and neglect of other groups}

Caution must be exercised in comparing the abuse experiences of people with intellectual disability with other groups who are marginalised. The life path and experiences of adults with intellectual disability who live in formal disability services is considerably different than that of children, women and elderly people (Stanley et al., 1999). Their experience of discrimination and marginalisation is different, and their historical experience of isolation and segregation is certainly different. However, there is value in drawing from these bodies of research to develop a more robust understanding of the features of emotional and psychological abuse and neglect of people with intellectual disability, particularly the focus on resilience from the women's abuse field and the shared conception of the damage that emotional abuse does to children.

\section{Children}

There is a considerable literature addressing the emotional and psychological abuse of children. One of the primary benefits of this literature when applied to people with intellectual disability is its focus on the development of clear definitions and criteria for identifying emotional and psychological abuse.

The child abuse literature is fairly consistent with regard to definitions of emotional and psychological abuse. Several authors (Iwaniec et al., 2006; Tucci and Goddard, 2003) draw from the work of key researchers Garbarino, Guttman and Seely (cited in Tucci and Goddard, 2003), Glaser (2002) and O'Hagan (1993) in identifying the critical elements of emotional and psychological abuse. Drawing together the findings of these seminal writers, the key features of working definitions of emotional and psychological child abuse include rejecting, isolating, terrorising, ignoring, corrupting, conditional parenting, insecure attachment, denigration and emotional unavailability.

The child abuse literature contains a far stronger emphasis on the impact, outcomes and damage done to children by this form of abuse than does the literature in either the disability or elder fields. There is consensus in the literature that the impacts of emotional and psychological abuse and neglect on children can be severe, far reaching and have a myriad of consequences (Glaser, 2002; Kairys et al., 2002; Vieth, 2004), all centring around the damage done to the child's sense of self and their understanding of the ways in which they relate to the people and world around them.

\section{Older people}

A considerable volume of work has been written on the issue of elder abuse, predominantly focusing on the domestic sphere, and the interaction between elders and carers (Slater, 2000; Biggs et al., 1995; Bennett et al., 1997). Elder abuse writers appear to find more common ground with the women's abuse and domestic violence literature, arising from a concern that elderly people and children occupy very different social, political and structural places in society. Several researchers have noted that both elder abuse and child abuse paradigms have been constructed by professionals, with the aim of protecting people and "solving" the problem. They have not grown from a grass roots, feminist perspective, as has the domestic violence and women's abuse literature (Bennett et al., 1997). Abuse of people with intellectual disability clearly falls into this category, and it is important to note the need for grass roots involvement in defining, recognising and designing responses to abuse and neglect.

\section{Women}

The literature on emotional and psychological abuse and neglect of women is almost wholly within the domestic sphere. The bulk of the literature relates to spouse abuse, or abuse within intimate relationships (Champagne, 1999; Barile, 2002). As with the elder abuse field, there is a considerable volume of material which focuses on the defining and understanding 
of emotional and psychological abuse, and a much greater focus on applied research, resources and strategies for women to first extract themselves from its grip, and second to recover from it. Here the literature differs from the other areas - the disability, child and elder abuse fields focus much more strongly on identifying potential impacts of abuse, and comparatively little on how people can recover from the experience.

Packota (2000, p. 4) proposes considering emotional and psychological abuse on a continuum. On one end are isolated hurtful behaviours that may occur in any relationship; at the other end is "pervasive, one-sided, severe psychological torture". McKinnon (2008) also subscribes to this view, and draws a useful distinction between emotional and psychological abuse in the domestic violence arena. She contends that there is significant overlap between verbal abuse, emotional abuse and psychological abuse, but that they are three categories of abuse. This distinction between emotional and psychological abuse, in which psychological abuse represents a deeper, longer term, power conflicted set of circumstances in which a person's sense of self and social competence is threatened may be very useful to our research. It may help to understand how long-term institutional practices help to create a "client", and to create a set of circumstances in which the continued mistreatment of people with intellectual disability is almost inevitable.

\section{Policy and practice responses to emotional and psychological abuse and neglect}

Our policy review was concentrated on the Australian context, due to the focus of the study. A range of definitions of emotional and psychological abuse were found within both government and non-government policy documents, often short and non-specific in nature. This may be a reflection of the very difficult ethical and moral judgements that practitioners need to make in taking action when abuse is suspected (Brown, 1999). The fact that so many policy documents seem to share a definition may also, however, reflect the fact that little attention has been paid to this form of abuse in our disability sector to date.

A consequence of the inclusion of emotional and psychological abuse and neglect as part of the "suite" of abuses experienced by people with intellectual disability is that definitions are frequently short, both in words and detail. In the case of abuses that are often subtle, and intimately bound up in relations of power, such definitions may not adequately explain these experiences. Adequate explanation and understanding of abuse, we would argue, is the foundation from which effective action against maltreatment can then be based.

The dominant definitions in use emphasise individual acts of maltreatment. For instance, the definition of emotional abuse most commonly used in the Australian policy context:

Verbal assaults, threats of maltreatment, harassment, humiliation or intimidation, or failure to interact with a person or to acknowledge that person's existence. This may also include denying cultural or religious needs and preferences (Australian Disability Abuse and Neglect Hotline, 2009).

Neglect is reflected broadly in Australian policy and resource documents, and based around action or lack of action. It is most often represented in ways similar to "a failure to provide the basic physical and emotional necessities of life" (Disability Services Queensland (DSQ), 2007a, p. 8) or "the failure to provide adequate support, food, shelter, clothing or hygienic living conditions"' (Verick and Fullwood, 1998, p. 10.6.1).

In the context of service provision in Australia, such definitions are contained in policy, which in turn sits inside Disability Services Standards, adherence to which are nominally monitored (either internally or externally, depending on the state). This definition is an output of service frameworks, which place heavy emphasis on response to individual instances of maltreatment, and considerably less emphasis on prevention and early intervention in potentially abusive situations.

Other jurisdictions take a broader view of abuse policy than does Australia, placing it within a context of prevention, protection and multi-agency cooperation in responding to incidents (such as the UK Government No Secrets guidelines on protecting vulnerable adults from abuse (UK Department of Health and UK Home Office, 2000) and In Safe Hands 
(Welsh Assembly Government, 2000), the Welsh equivalent policy framework). Australian high-level abuse policy sets a framework of prevention, response and protection, but does not contain a multi-agency focus, having more a focus on containment and resolution of individual problems. The impact of the UNCRPD on abuse policy in the Australian domestic context remains to be seen.

Several practice and policy manuals reviewed in the course of this study contain checklists of physical indicators and behavioural signs of abuse (Bhargava, 2006; Page et al., 2002; DSQ, 2007b). There is a difficult balance between providing guidance for staff who may not perceive emotional or psychological harm without some assistance and narrowing the conception of emotional and psychological harm to such an extent that service recognition of it is formulaic and workers only respond to harm which is "on the list". The work of Page et al. (2002) includes an analysis of the environmental and cultural causative factors of abuse and neglect, which places a checklist in a broader context. Without this context, there is a considerable danger that workers may not have the knowledge and skills needed to interpret behavioural or physical indicators of abuse effectively, or to take appropriate action if they do.

An evolving framework for understanding emotional and psychological abuse and neglect of people with intellectual disability

Opinion on expansive definitions of abuse and neglect is divided in the literature. Some authors consider the range of abusive behaviours to be virtually inexhaustible, and contend that there is risk in developing a "checklist" of abusive behaviours, particularly in the case of abuses that may not have tangible indicators (Conway et al., 1996; Brown, 1997; Vieth, 2004). This may encourage formulaic responses - if a worker discovers that an abusive behaviour is not on the list, for example, then there is a risk they will not take action to end the abuse occurring in the life of the person with intellectual disability. As Brown (1999, p. 97) maintains:

it is the identification of abuse within the context of a number of more or less closed interlocking systems that is problematical rather than the definition.

There is also a body of research which builds a case that the disability sector focuses far more on responding to abuse once it has already occurred than on preventing it from occurring in the first place (White et al., 2003; Marsland et al., 2007; Page et al., 2002). In part, this may be due to a lack of capacity to recognise the more subtle signs of abuse. WWDA (2007) points out that any definition needs to incorporate the structural roots of violence so that analysis can be meaningful, and Bright (1999, p. 128) cautions that:

Producing definitions, while useful to those whose work involves them in writing policies and procedures, may have the effect of disguising the routine indignity that many people may be exposed to day by day, throughout the day and possibly night time too.

However, in order to better understand a comparatively under researched and little considered form of maltreatment, we feel there is value in developing a framework for understanding emotional and psychological abuse and neglect which goes into some detail in describing the sorts of behaviours and interactions which can occur when it is inflicted, and which is tested against the experiences of people who have experienced this sort of abuse and neglect. In a climate in which gross physical and sexual abuses of people with intellectual disability are often poorly responded to (Sobsey, 1994; Chenoweth, 1995; Fawcett, 2008; Howe, 2000), there may be even less hope of subtle abuses being recognised and acted upon without clear and direct identification through definitions which have been developed with people who have lived through the experiences themselves.

The literature on abuse and neglect of people with disability, children, older people and women was used to scaffold a framework for understanding emotional and psychological abuse and neglect, and this was applied in our study. The framework provides detail on a possible range of abuses, which could occur in this domain, and allows for the classification of those abuses into key theme areas. Figure 1 shows some of the grouped features of this form of abuse. It has been adapted by Robinson (2010), from the work of Kovener (2000). 


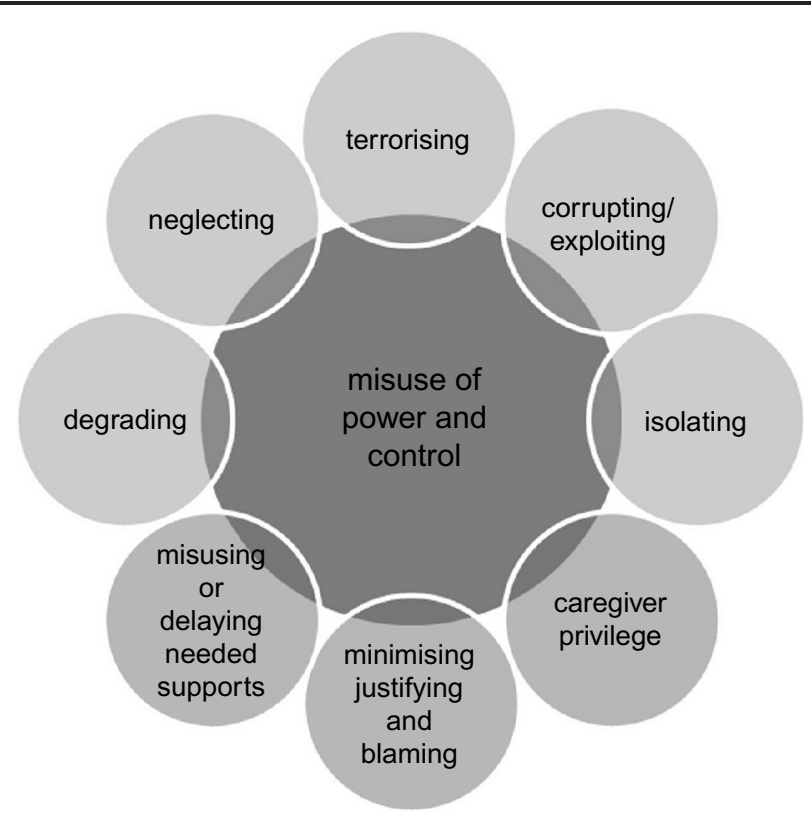

At the centre of the framework is the misuse of power and control. The key assumption of the framework is that the experience of emotional and psychological abuse and neglect in disability accommodation services is intimately connected to abuses of power and control. This assumption builds from the literature on power relations, and on conceptions and constructions of disability which influence service design and delivery as discussed above (Marsland et al., 2007; Lindemann Nelson, 2001; Chenoweth, 1995; Mandeville and Hanson, 2000; Robinson and Chenoweth, 2011a, b). This body of work consistently holds that imbalances of power and control are structured into disability services, creating a climate in which abuse and neglect is likely to occur.

Within this climate or systemic environment, the experience of emotional and psychological abuse is conceived as encompassing several characteristics. These characteristics, or themes, have been drawn from the child, women's, elder and disability abuse literature (Tucci and Goddard, 2003; Glaser, 2002; O'Hagan, 1995; Biggs et al., 1995; Champagne, 1999; Kovener, 2000). They are:

- Terrorising. Coercing; threatening to hurt; frightening; intimidating; withholding basic support and rights; terminating relationship and leaving the person unattended; reporting non-compliance with a programme; using more intrusive equipment; using consequences and punishments to gain compliant behaviour; pressuring the person to engage in fraud or other crimes.

- Corrupting/exploiting. Socialising a person into accepting ideas or behaviour which oppose legal standards; using a person for advantage or profit; training a person to serve the interests of the abuser.

- Isolating. Controlling access to friends, family and neighbours; controlling access to phone, TV, news; limiting employment possibilities because of caregiver schedule(s); discouraging contact with the case manager or advocate.

- Degrading. Punishing or ridiculing; refusing to speak; ignoring requests; ignoring the person; harassing; humiliating; ridiculing the person's culture, traditions, religion; ridiculing personal tastes; enforcing a negative reinforcement or behaviour programme the person does not consent to.

- Neglecting. Failing to provide nurturance; failing to provide stimulation. 
While the themes of degrading, terrorising, corrupting/exploiting and isolating are common to all abused groups and environments, the themes of caregiver privilege, minimising, justifying and blaming and withholding, misusing and delaying needed supports are used here specifically in relation to the "care" environment.

- Caregiver privilege. Treating the person like a child or servant; making unilateral decisions; defining narrow, limiting roles and responsibilities; providing care in a way to accentuate the person's dependence and vulnerability; giving an opinion as if it were the person's opinion; denying the person the right to privacy; ignoring; discouraging; prohibiting the exercise of full capabilities.

- Minimising, justifying and blaming. Denying or making light of abuse; denying physical and emotional pain; justifying rules that limit autonomy, dignity and relationships for program's operational efficiency; excusing abuse as behaviour management; excusing abuse as caregiver stress; blaming the disability for the abuse; saying the person is not a "good reporter" of abuse.

- Withholding, misusing or delaying needed supports. Using medication to sedate the person for agency convenience; ignoring equipment safety requirements; breaking or not fixing adaptive equipment; refusing to use or destroying communication devices; withdrawing care or equipment to immobilise the person; using equipment to torture the person.

The child abuse literature also offers categories of rejecting and denying emotional responsiveness (Tucci and Goddard, 2003; O'Hagan, 1995). In the disability accommodation services setting, these have been incorporated into the concepts of caregiver privilege, and minimising, justifying and blaming.

Neglect is included in the framework, but conceptually needs to be seen as an integral part of emotional and psychological abuse, where the omission of action may be as damaging as the commission of abusive action (as in the case of a person who does not learn to use a communication device due to lack of staff assistance to do so). Neglect can be seen in (at least) two ways, as a failure to provide nurturing or emotional support, and as a failure to provide stimulation.

\section{Discussion}

This framework for understanding emotional and psychological abuse has been tested against the lived experiences of people with intellectual disability who participated in the research as part of the research method, narrative collage (Robinson, 2010). It is critical that a definition or framework has resonance with the lived experience of people with intellectual disability in order to accurately reflect the sorts of abuse that they endure. There are weighty cultural, environmental and organisational pressures brought to bear on the experience of abuse which influence both the way in which it is experienced and the way in which it is responded to. Some of these pressures influence the descriptions of abuse, and may result in definitions of abuse that are dominated by a professionalised understanding of abuse, with service responses to allegations of assault uppermost in mind, rather than an understanding which is developed from lived experience. As Manthorpe and Stanley (1999, p. 115) note in their work with people with intellectual disability about their perceptions of abuse:

listening to individual's voices, however, reminds us that abuse is often subjective.

While several domains within the framework are common to maltreatment of other groups, the framework further develops the categories of maltreatment into domains experienced in the service context by people with intellectual disability, which are not shared by other groups (children, women, elders). From this flow several implications for policy and practice. Questions are raised about how we might support staff who work with people with intellectual disability to better recognise and respond to both the preconditions and the experience of emotional and psychological abuse and neglect. The framework may prove a useful training tool to assist disability support workers, managers, family members and other supports such as advocates to better understand this form of abuse and neglect and how they can 
recognise and respond to it. It is important that they are also provided with an analysis of the role of systems and social structures in this abuse and neglect, as part of the development of their own ethical competence (Doel et al., 2010). An adapted form may be of use to people with intellectual disability in training and education.

A more complex construction of emotional and psychological abuse and neglect may also inform the development of policy for disability accommodation services. This may assist policy makers to recognise, and hence to reflect in policy, both the potential range of actions which can occur and the central place of systems and social structures in this form of abuse and neglect. A broadened view of the range of behaviours which can be viewed within the service frame as abusive, rather than poor practice or human resource problems, would stand to change the way we recognise subtle and ongoing abuse and maltreatment. For example, the experiences of being ignored, being discouraged, or having your life limited due to workers rosters are far more likely to occur on a regular or repeated basis than as a critical incident, to not meet the criteria for reporting that are common to disability services, and to result in a lived experience which, while it may be analogous to "death by a thousand cuts", may be unseen in a policy context.

An important area for further research concerns the experience of subtle abuse, and emotional and psychological maltreatment in self-directed support environments. The international growth of self-direction as a support philosophy has not been matched by policy and practice that articulates prevention and response around subtle and hard to express forms of abuse and neglect. For those people who are reliant on family and paid staff to mediate their relationships with others, both opportunities and risks are brought into high contrast when considering emotional abuse and self-directed supports.

\section{Conclusion}

We hope that this evolving framework provides a more rounded range of behaviours against which to map people's experiences. There is no intent to be prescriptive, nor limiting of the myriad emotional and psychological abuses and neglects that are unfortunately possible in the lives of people with intellectual disability.

We had several goals in developing this framework. First, we sought to apply the knowledge gleaned from the many scholars who preceded us to our study and to take learning from the child, elder and women's abuse domains into the disability abuse field. Second, we felt it important to ensure that the draft framework had resonance with people's lived experience(s) - both the experiences of key participants in the study (those with intellectual disability) and through seeking the views of their advocates and family members about the appropriateness and "on the mark" quality of the framework. Third, we used the framework as a research tool, and thematically analysed research data against the core concepts of the framework. This process added some detail to the framework and confirmed its basic integrity. However, this small study has only piloted the framework, and without doubt more work is needed to establish its rigour under a range of applications. Finally, we are in the process of sharing the framework with policy makers, service providers, disability advocates and people with disability as part of broader work to raise awareness and promote alternative responses to emotional and psychological harm.

\section{References}

Australian Disability Abuse and Neglect Hotline (2009), "What is abuse and neglect?", available at: www.disabilityhotline.org/abuse.html\#psychological_abuse (accessed 29 January 2009).

Barile, M. (2002), "Individual-systemic violence: disabled women's standpoint", Journal of International Women's Studies, Vol. 4 No. 1, pp. 1-14.

Bennett, G., Kingston, P. and Penhale, B. (1997), The Dimensions of Elder Abuse: Perspectives for Practitioners, Macmillan, Basingstoke.

Bhargava, D. (2006), Identification of Abuse, Sydney (unpublished work). 
Biggs, S., Phillipson, C. and Kingston, P. (1995), Elder Abuse in Perspective, Open University Press, Buckingham

Bright, L. (1999), "Elder abuse in care and nursing settings: detection and prevention", in Slater, P. and Eastman, M. (Eds), Elder Abuse: Critical Issues in Policy and Practice, Age Concern England, London.

Brown, H. (1999), "Abuse of people with learning disabilities: layers of concern and analysis", in Stanley, N., Manthorpe, J. and Penhale, B. (Eds), Institutional Abuse: Perspectives Across the Life Course, Routledge, London.

Brown, R.I. (1997), Quality of Life for People with Disabilities: Models, Research and Practice, Stanley Thomas, Cheltenham.

Champagne, C. (1999), "Wearing her down: understanding and responding to emotional abuse", available at: www.womanabuseprevention.com/html/wearing_her_down.html (accessed 6 November 2006).

Chenoweth, L. (1995), "The mask of benevolence: cultures of violence and people with disabilities", in Bessant, J., Carrington, K. and Cook, S. (Eds), Cultures of Crime and Violence: The Australian Experience, La Trobe University Press, Melbourne.

Conway, R.N.F. (1994), "Abuse and intellectual disability: a potential link or an inescapable reality?", Australian and New Zealand Journal of Developmental Disabilities, Vol. 19 No. 3, pp. 165-71.

Conway, R.N.F., Bergin, L. and Thornton, K. (1996), Abuse and Adults with Intellectual Disability Living in Residential Services, Office of Disability, Canberra.

Disability Services Queensland (2007a), Portfolio Policy Statement: Preventing and Responding to Abuse, Assault and Neglect of People with a Disability, Queensland Government, Brisbane.

Disability Services Queensland (2007b), Growing Stronger: Investing in a Better Disability Service System, Queensland Government, Brisbane.

Doel, M., Allmark, P., Conway, P., Cowburn, M., Flynn, M., Nelson, P. and Tod, A. (2010), "Professional boundaries: crossing a line or entering the shadows?", British Journal of Social Work, Vol. 40, pp. 1866-99.

Fawcett, B. (2008), "Disability and violence", in Fawcett, B. and Waugh, F. (Eds), Addressing Violence, Abuse and Oppression: Debates and Challenges, Routledge, Abingdon.

Glaser, D. (2002), "Emotional abuse and neglect (psychological maltreatment): a conceptual framework", Child Abuse and Neglect, Vol. 26 No. 10, pp. 697-714.

Horne, S., Merz, T.A. and Merz, D.P. (2001), "Disability and emotional abuse: mental health consequences and social implications", Journal of Emotional Abuse, Vol. 2 No. 4, pp. 39-60.

Howe, K. (2000), Violence Against Women with Disabilities: A Review of the Literature, Women With Disabilities Australia, Melbourne.

Iwaniec, D., Larkin, E. and Higgins, S. (2006), "Research review: risk and resilience in cases of emotional abuse", Child and Family Social Work, Vol. 11, pp. 73-82.

Jenkins, R. and Davies, R. (2006), "Neglect of people with intellectual disabilities: a failure to act?", Journal of Intellectual Disability, Vol. 10 No. 1, pp. 35-45.

Kairys, S.W., Johnson, C.F. and Committee on Child Abuse and Neglect (2002), "The psychological maltreatment of children - technical report", Pediatrics, Vol. 109, pp. 68-71.

Kovener, M. (2000), "Collaborating to serve victims of crime in Denver: victim service 2000", Impact, Vol. 3 No. 3, p. 23.

Lindemann Nelson, H. (2001), Damaged Identities, Narrative Repair, Cornell University Press, New York, NY.

McKinnon, L. (2008), "Hurting without hitting: non-physical contact forms of abuse", Stakeholder Paper 4, Australian Domestic Violence Clearinghouse, Sydney, pp. 1-15.

Mandeville, H. and Hanson, M. (2000), "Understanding caregiver abuse as domestic violence: systemic change in Wisconsin", Impact, Vol. 13 No. 3, pp. 14-15, 27. 
Manthorpe, J. and Stanley, N. (1999), "Conclusion: shifting the focus, from 'bad apples' to users' rights", in Stanley, N., Manthorpe, J. and Penhale, B. (Eds), Institutional Abuse: Perspectives Across the Life Course, Routledge, London.

Marsland, D., Oakes, P. and White, C. (2007), "Abuse in care? The identification of early indicators of the abuse of people with learning disabilities in residential settings", Journal of Adult Protection, Vol. 9 No. 4, pp. 6-20.

Nosek, M., Clubb Foley, C., Hughes, R.B. and Howland, C. (2001a), "Vulnerabilities for abuse among women with disabilities", Sexuality and Disability, Vol. 19 No. 3, pp. 177-90.

Nosek, M., Howland, C. and Hughes, R.B. (2001b), "The investigation of abuse and women with disabilities: going beyond assumptions", Violence Against Women, Vol. 7, pp. 477-99.

O'Hagan, K. (1993), Emotional and Psychological Abuse of Children, Open University Press, Buckingham.

O'Hagan, K. (1995), "Emotional and psychological abuse: problems of definition", Child Abuse and Neglect, Vol. 19 No. 4, pp. 449-61.

Packota, V.J. (2000), Emotional Abuse of Women by Their Intimate Partners: A Literature Review, Education Wife Assault, Toronto.

Page, S., Lane, P. and Kempin, G. (2002), Abuse Prevention Strategies in Specialist Disability Services, National Disability Administrators, Canberra.

Penhale, B. (1999), "Introduction", in Stanley, N., Manthorpe, J. and Penhale, B. (Eds), Institutional Abuse: Perspectives Across the Life Course, Routledge, London.

Robinson, S. (2010), "Insult and injury: a narrative approach to understanding the emotional and psychological abuse and neglect of people with intellectual disability living in disability accommodation services", PhD thesis, Griffith University, Brisbane.

Robinson, S. and Chenoweth, L. (2011a), "Front and centre: using narrative collage to privilege the voice of people with intellectual disability in research about abuse and neglect", Qualitative Q1 Methodology, Under review.

Robinson, S. and Chenoweth, L. (2011b), "Preventing abuse in accommodation services: from procedural response to protective cultures", Journal of Intellectual Disability, Vol. 15 No. 1, pp. 63-74.

Saxton, M. (Ed.) (2009), Sticks and Stones: Disabled People's Stories of Abuse, Defiance and Resilience, World Institute on Disability, Oakland, CA.

Slater, P. (2000), "Elder abuse and user involvement: strategic components", Journal of Adult Protection, Vol. 2 No. 2, pp. 18-28.

Sobsey, D. (1994), Violence and Abuse in the Lives of People with Disabilities: The End of Silent Acceptance?, Paul H. Brookes, Baltimore, MD.

Stanley, N., Manthorpe, J. and Penhale, B. (Eds) (1999), Institutional Abuse: Perspectives Across the Life Course, Routledge, London.

Ticoll, M. (1994), Violence and People with Disabilities: A Review of the Literature, The Roeher Institute, Toronto.

Tucci, J. and Goddard, C. (2003), "Emotional abuse of children: a study of the narratives in protective assessment in intervention", paper presented at Many Voices, Many Choices: Ninth Australasian Conference on Child Abuse and Neglect, Australian Childhood Foundation, Sydney.

UK Department of Health and UK Home Office (2000), No Secrets: Guidance on Developing and Implementing Multi-agency Policies and Procedures to Protect Vulnerable Adults from Abuse, UK Department of Health and Home Office, London.

Verick, M. and Fullwood, D. (1998), Standards in Action Manual, Ageing and Disability Department NSW, Sydney.

Vieth, V. (2004), "When words hurt: investigating and proving a case of psychological maltreatment", Reasonable Efforts, Vol. 2 No. 1, available at: www.ndaa.org/publications/newsletters/reasonable_ efforts_volume_2_number_1_2004.html (accessed 14 May 2007). 
Welsh Assembly Government (2000), In Safe Hands: Implementing Adult Protection Procedures in 628 Wales, Welsh Assembly Government, Cardiff.

Westcott, H. (1993), Abuse of Children and Adults with Disabilities, National Society for the Prevention of 630 Cruelty to Children, London.

White, C., Holland, E., Marsland, D. and Oakes, P. (2003), "The identification of environments and cultures that promote the abuse of people with intellectual disabilities: a review of the literature", Journal of Applied Research in Intellectual Disabilities, Vol. 19, pp. 1-9.

WWDA (2007), Forgotten Sisters: A Global Review of Violence Against Women with Disabilities, Women with Disabilities Australia, Hobart.

\section{Further reading}

O'Hagan, K. (2006), Identifying Emotional and Psychological Abuse: A Guide for Childcare 642 Professionals, Open University Press, Berkshire.

\section{Corresponding author}

Sally Robinson can be contacted at: sally.robinson@scu.edu.au 